\title{
Identifying different types of bulking in an activated sludge system through quantitative image analysis
}

\author{
D.P. Mesquita a , A.L. Amaral ${ }^{\mathrm{a}, \mathrm{b}}$, E.C. Ferreira ${ }^{\mathrm{a}, *}$ \\ a IBB-Institute for Biotechnology and Bioengineering, Centre of Biological Engineering, Universidade do Minho, Campus de Gualtar, 4710-057 Braga, Portugal \\ ${ }^{\mathrm{b}}$ Instituto Superior de Engenharia de Coimbra, Instituto Politécnico de Coimbra, Rua Pedro Nunes, Quinta da Nora, 3030-199 Coimbra, Portugal
}

\section{A R T I C L E I N F O}

\section{Article history:}

Received 24 February 2011

Received in revised form 11 July 2011

Accepted 13 July 2011

Available online 12 August 2011

\section{Keywords:}

Activated sludge

Image analysis

Physiological status

Sludge volume index

Total suspended solids

\begin{abstract}
A B S T R A C T
The present study proposes an image analysis methodology for the identification of different types of disturbances in wastewater treatment activated sludge systems. Up to date, most reported image analysis methodologies have been used in activated sludge processes with the aim of filamentous bulking detection, however, other disturbances could be foreseen in wastewater treatment plants. Such disturbances can lead to fluctuations in the biomass contents, affecting the mixed liquor suspended solids (MLSS), and in the sludge settling ability, affecting the sludge volume index (SVI). Therefore, this work focuses on predicting the MLSS and SVI parameters for different types of disturbances affecting an activated sludge system. Four experiments were conducted simulating filamentous bulking, zoogleal or viscous bulking, pinpoint floc formation, and normal operating conditions. Alongside the MLSS and SVI determination, the aggregated and filamentous biomass contents and morphology were studied as well as the biomass Gram and viability status, by means of image analysis.
\end{abstract}

다 2011 Elsevier Ltd. All rights reserved.

\section{Introduction}

Typically, an activated sludge system is a continuous biological process extensively used in wastewater treatment plants (WWTPs). As most biological processes, activated sludge systems are sensitive to sudden changes leading to large economical and environmental consequences. In an attempt to monitor and control the biological process, the past decades have witnessed a significant growth of activated sludge inspection by means of optical microscopy. In this context, research increased the knowledge regarding the determination of activated sludge structure, including morphological, physical, and chemical parameters which are closely related to solid-liquid separation (Li and Ganczarczyk, 1987; Urbain et al., 1993; Wilén et al., 2003; Jin et al., 2004; Liao et al., 2006; Li and Yang, 2007).

It is known that activated sludge is affected by common malfunctions such as: formation of pinpoint flocs (PP); filamentous bulking; and viscous or zoogleal bulking (ZB). PP phenomena are characterized by the formation of small and mechanically fragile activated sludge flocs, presenting low settling properties, formed by floc-forming bacteria and lacking the filamentous bacteria backbone (Jenkins et al., 2003). As the majority of these flocs does not settle, the sludge volume index (SVI) parameter is not strongly affected by the formation of PP. However, in such instances, the clar-

\footnotetext{
* Corresponding author. Tel.: +351 253604 407; fax: +351 253678986.

E-mail address: ecferreira@deb.uminho.pt (E.C. Ferreira).
}

ifier's supernatant presents high solids concentration, thus leading to the biomass washout from the reactor, and a correspondent decrease on the reactor solids. Filamentous organisms are an essential part of the floc population in an activated sludge process, forming the backbone to which floc-forming bacteria adhere. However, filamentous bulking may take place when filamentous bacteria overgrow, leading to poor sludge settling ability and poor thickening characteristics of the sludge. Filamentous bulking is one of the most studied problems regarding activated sludge (Eikelboom, 2000; Jenkins et al., 2003; Martins et al., 2004; Schuler and Jassby, 2007). Viscous or ZB is caused by an excessive amount of extracellular polysaccharides (EPS), and has been shown to have a negative effect on the biomass thickening and compaction due to the water-retentive nature of EPS, making the activated sludge flocs density closer to that of the surrounding water, thus increasing the SVI (Novak et al., 1993; Jobbágy et al., 2002; Jin et al., 2003; Jenkins et al., 2003; Peng et al., 2003). In extreme, severe instances of viscous bulking were shown to result in the absence of solids separation (Jenkins et al., 2003).

Microscopy observations are becoming ever more important methods to monitor and control activated sludge systems and, as a result of that, these techniques are becoming widespread for the characterization of activated sludge microbial aggregates (Andreadakis, 1993; Barbusinski and Koscielniak, 1995). Furthermore, the association of image processing and analysis methodologies with microscopy visualization allows an accurate evaluation of the activated sludge status (Li and Ganczarczyk, 1991; Grijspeerdt and Verstraete, 1997). Several authors (da Motta et al., 2002; 
Cenens et al., 2002; Amaral and Ferreira, 2005; Jenné et al., 2006; Mesquita et al., 2009a,b; Arelli et al., 2009) have already proposed an array of image analysis procedures to characterize and relate operating parameters, such as the SVI, with biomass structure of activated sludge systems, mainly in terms of aggregated and filamentous biomass. However, up to the present, most studies relating SVI and sludge morphological properties have been focused on filamentous bulking conditions solely (da Motta et al., 2002; Amaral and Ferreira, 2005). Moreover, the emphasis of such studies has been focused on the biomass contents and morphology, and little attention has been given to the Gram type and physiological status (viable or damaged) of such biomass.

Gram staining can be problematic regarding large and dense flocs which may not decolorize correctly, and could be problematic for Gram status identification through image analysis procedures, aggravated by the narrow width of the filamentous bacteria (inducing color distortion effects on the image acquisition) and the color proximity of Gram (-) and Gram (+) bacteria (pink and violet, respectively). The development of novel fluorescent nucleic acid binding dyes, such as SYTO 9 and hexidium iodide (HI), allows the assessment of Gram status by differential absorption through bacterial cell walls and without fixative methods. In this sense, the use of such fluorescent dyes may provide a robust, objective, and rapid alternative to traditional Gram staining in wastewater systems (Foster et al., 2002). For viability detection, fluorescent nucleic acid binding dyes such as SYTO 9 and propidium iodide (PI), differing in their ability to penetrate healthy bacterial cells, differentiate live from dead or damaged cells by detecting cell membrane integrity, even in a mixed population containing a broad range of bacterial types (Invitrogen Molecular Probes, 2004).

The present study aims to identify and quantify aggregated and filamentous biomass from a lab-scale activated sludge system using image processing and analysis procedures in bright field microscopy. The biomass composition on Gram $(+)$ and Gram $(-)$ bacteria, as well as viable and damaged bacteria, was also evaluated by image analysis coupled to fluorescent staining. Finally, the obtained image analysis data were used to identify activated sludge malfunctions (PP formation, filamentous bulking and ZB), and further correlated with SVI and mixed liquor suspended solids (MLSS) parameters.

\section{Materials and methods}

\subsection{Experimental setup}

Experiments were carried out in a lab-scale activated sludge system composed by a $17 \mathrm{~L}$ reactor and a $2.5 \mathrm{~L}$ settler. The system was equipped with feed pumps, air supply at the bottom of the reactor, and sensor apparatus ( $\mathrm{pH}$ meter with a control pump and a dissolved oxygen probe). The sludge recirculation was performed from the settler to the reactor by a pneumatic pump.

\subsection{Synthetic wastewater composition and sludge characteristics}

The system was fed with a synthetic medium prepared with the following composition $\left(\mathrm{mg} \mathrm{L}^{-1}\right)$ : $\mathrm{NaCH}_{3} \mathrm{COO} \cdot 3 \mathrm{H}_{2} \mathrm{O}, 2073$; $\left(\mathrm{NH}_{4}\right)_{2} \mathrm{SO}_{4}, 140 ; \mathrm{MgSO}_{4} \cdot 7 \mathrm{H}_{2} \mathrm{O}, 25 ; \mathrm{KH}_{2} \mathrm{PO}_{4}, 44 ; \mathrm{K}_{2} \mathrm{HPO}_{4} \cdot 2 \mathrm{H}_{2} \mathrm{O}, 59$; $\mathrm{CaCl}_{2} \cdot 2 \mathrm{H}_{2} \mathrm{O}, 30 ; \mathrm{FeCl}_{3} \cdot 6 \mathrm{H}_{2} \mathrm{O}, 18.8 ; \mathrm{NaHCO}_{3}, 105$, and diluted for a final COD of $1000 \mathrm{mg} \mathrm{L}^{-1}$. For biomass maintenance, a micronutrients solution was also added with the following composition $\left(\mathrm{mg} \mathrm{L}^{-1}\right): \mathrm{H}_{3} \mathrm{BO}_{3}, 50 ; \mathrm{ZnCl}_{2}, 50 ; \mathrm{CuI}_{2} \cdot \mathrm{H}_{2} \mathrm{O}, 40 ; \mathrm{MnCl}_{2}, \quad 20$; $\left(\mathrm{NH}_{4}\right)_{6} \mathrm{Mo}_{7} \mathrm{O}_{24} \cdot 4 \mathrm{H}_{2} \mathrm{O}, 55 ; \mathrm{AlCl}_{3}, 50 ; \mathrm{NiCl} \cdot 6 \mathrm{H}_{2} \mathrm{O}, 110$. The $\mathrm{pH}$ of the system was maintained at approximately 7 with a pH meter (Model 924001, Jenway Scientific, UK) and a control pump (Model BL 7916-BL 7917, Hanna Instruments, Woonsocket, RI, USA) that dosed $0.01 \mathrm{M} \mathrm{HCl}$ solution when the $\mathrm{pH}$ was above the set point. The temperature of the system was maintained at room temperature. The aerated tank was inoculated with activated sludge from a domestic wastewater treatment plant. An initial MLSS concentration of $4-6.5 \mathrm{~g} \mathrm{~L}^{-1}$ in the aerated tank was used. The study of the four conditions (filamentous bulking, PP formation, ZB, and normal conditions, NC) was sequentially conducted, and between each experiment, the system was re-inoculated with biomass to guarantee a rapid establishment of the new condition. Images obtained for each condition are presented in Fig. 1. For the filamentous bulking phenomena experiment (FB1 and FB2), the organic loading rate (OLR) was increased from 0.1 to $0.38 \mathrm{~kg} \mathrm{COD} \mathrm{kg}^{-1} \mathrm{MLSS} \mathrm{d}^{-1}$. The PP experiment was conducted decreasing the OLR from 0.2

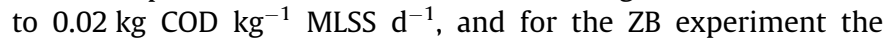
OLR was increased from 0.02 to $0.2 \mathrm{~kg} \mathrm{COD} \mathrm{kg}^{-1} \mathrm{MLSS} \mathrm{d}^{-1}$. Finally, the NC experiment was performed with an OLR ranging between 0.126 and $0.152 \mathrm{~kg} \mathrm{COD} \mathrm{kg}^{-1} \mathrm{MLSS} \mathrm{d}^{-1}$. For both filamentous and ZB conditions a solids retention time (SRT) of around $20 \mathrm{~d}$ was used, whereas for the PP and NC experiments the SRT was in average 0.8 and $0.5 \mathrm{~d}$, respectively.

\subsection{Off-line process monitoring}

During each experiment, MLSS measurements were conducted in accordance with the procedures described in Standard Methods (APHA, 1989). The biomass settling ability was measured through the determination of the SVI in a $1 \mathrm{~L}$ Imhoff cone, with the sludge height variation monitored for $30 \mathrm{~min}$. Whenever a low settling ability was detected (sludge volume higher than $250 \mathrm{~mL}$ ), a dSVI (diluted) was used (Jenkins et al., 2003; Amaral and Ferreira, 2005).

\subsection{Bright field image acquisition}

Periodic samples were taken from the aerated tank to assess the morphology of the activated sludge by bright field microscopy. A recalibrated micropipette with a sectioned tip at the end, with a large enough diameter to allow larger aggregates to flow, was used to deposit samples on slides. Three slides per sample were used, and for each slide a volume of $10 \mu \mathrm{L}$ was covered with a $20 \mathrm{~mm} \times 20 \mathrm{~mm}$ cover slip, for visualization and image acquisition. Images were acquired in the upper, middle and bottom of the slide in order to improve the representativeness of the microbial community in the system resulting in a total of 150 images ( $3 \times 50$ images/slide). The slides were examined by means of an Olympus BX51 optical microscope (Olympus, Tokyo, Japan), at $100 \times$ total magnification, coupled with an Olympus DP25 camera (Olympus, Tokyo, Japan). Images were acquired at $1360 \times 1024$ pixels and 8-bit format through the commercial software Cell ${ }^{\wedge} \mathrm{B}$ (Olympus, Tokyo, Japan).

\subsection{Staining procedures}

The Live/Dead BacLight bacterial viability kit was used to differentiate viable and damaged bacteria (Molecular Probes, Eugene, Oregon, USA). The kit utilizes a mixture of SYTO 9 green-fluorescent nucleic acid stain and a red-fluorescent nucleic acid stain, PI. Viable bacteria are stained by SYTO 9 and damaged bacteria are stained by PI.

The Live Baclight bacterial Gram stain allows classifying bacteria as Gram (+) or Gram (-) without the use of fixatives (Molecular Probes, Eugene, Oregon, USA). This kit utilizes a mixture of SYTO 9 green-fluorescent nucleic acid stain and a red-fluorescent nucleic acid stain, HI. Gram (-) bacteria are stained by SYTO 9 and Gram (+) bacteria are stained by $\mathrm{HI}$.

The staining protocol is described in Boulos et al. (1999) and next are stated some adjustments. First, a sterile solution of 

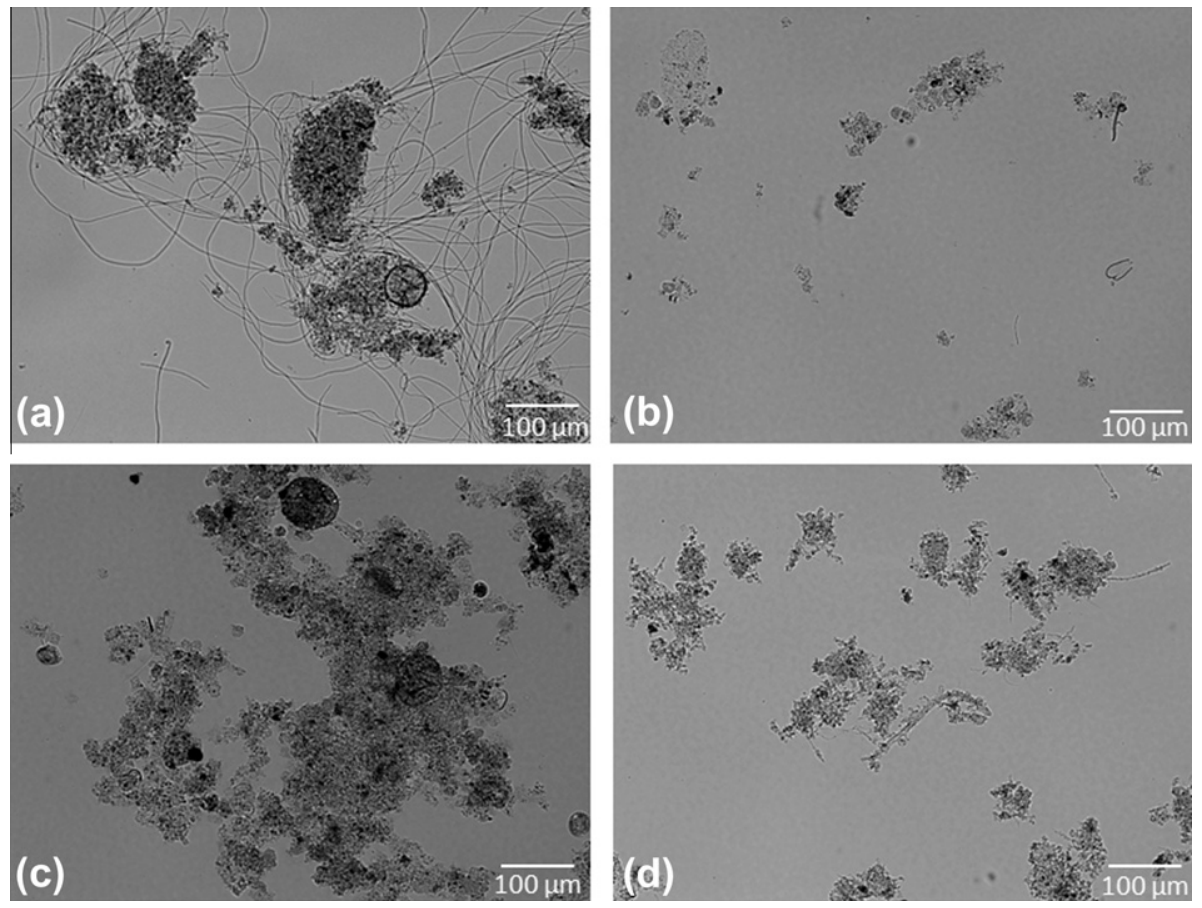

Fig. 1. (a) Filamentous bulking, (b) pinpoint flocs, (c) zoogleal bulking and (d) normal flocs.

$0.85 \% \mathrm{NaCl}$ was prepared. For the staining solutions, $1.5 \mu \mathrm{L}$ of each dye was put into $5 \mathrm{~mL}$ of the $\mathrm{NaCl}$ solution and the tube was wrapped with aluminum foil and protected from light. A volume of $100 \mu \mathrm{L}$ of undiluted biomass suspension from the reactor was mixed with $50 \mu \mathrm{L}$ of staining solution and incubated in darkness for $15 \mathrm{~min}$ at room temperature. A preliminary experiment allowed concluding that this dye concentration was sufficient for staining the overall bacteria population. The bacteria population was then visualized through epifluorescence microscopy.

\subsection{Epifluorescence microscopy}

Periodic samples were taken from the aerated tank to assess the physiology of the activated sludge by staining and epifluorescence microscopy. As above, a recalibrated micropipette with a sectioned tip at the end was used to deposit samples on slides. Two slides per sample were used, and for each slide a volume of $10 \mu \mathrm{L}$ of the stained sludge samples was covered with a $20 \mathrm{~mm} \times 20 \mathrm{~mm}$ cover slip, for visualization and image acquisition. Again, images were acquired in the upper, middle and bottom of the slide resulting in a total of 100 images $(2 \times 50$ images/slide). The slides were examined by means of an epifluorescence microscope Olympus BX51 (Olympus, Tokyo, Japan) at $200 \times$ total magnification. Two long pass filters were used, one in the green wavelength range with an excitation bandpass of $470-490 \mathrm{~nm}$ and emission at $516 \mathrm{~nm}$, and a second filter in the red wavelength range with an excitation bandpass of $530-550 \mathrm{~nm}$ and emission at $591 \mathrm{~nm}$. Images were acquired at $1360 \times 1024$ pixels, and 24-bit RGB format ( 8 bit red, 8 bit green and 8 bit blue channels) through the commercial software Cell^ ${ }^{\wedge}$ (Olympus, Tokyo, Japan).

\subsection{Bright field image processing}

Bright field program was adapted from a previous version of Amaral (2003), developed in Matlab 7.3 (The Mathworks, Natick, USA) for the recognition of aggregates and filamentous bacteria in grayscale images. A more detailed description of the image pro- cessing methods can be found in Mesquita et al. (2010). The original grayscale image and the final binary images of aggregated and filaments biomass are presented in Fig. 2A.

\subsection{Fluorescence image processing}

Fluorescence program was also developed in Matlab 7.3 (The Mathworks, Natick, USA) to recognize and characterize the aggregated and filamentous biomass in the fluorescent images, extracting the green and the red channels, followed by background correction, image segmentation and aggregated and filamentous biomass recognition, for each of the above channels. The developed program allowed for the calculation of a fluorescence-based intensity image, for both green and red channels, directly correlated with the fluorescence of the aggregated and filamentous biomass. These images were subsequently used, regarding the filamentous biomass, to accurately identify the Gram and viability status of overlapping SYTO 9 and PI or HI stained cells. A schematic description of the image processing program is presented in Fig. 2B.

\subsection{Morphological and physiological parameters determination}

After the image processing step, a routine in Matlab 7.3 (The Mathworks, Natick, USA) was developed for parameters calculation. The segmentation of the bright field grayscale images allowed to automatically determine the aggregated and filamentous biomass morphology and contents. Aggregates were classified according to their size in (Eikelboom, 2000): small aggregates $\left(D_{\mathrm{eq}}<25 \mu \mathrm{m}\right)$; intermediate aggregates $\left(25<D_{\mathrm{eq}}<250 \mu \mathrm{m}\right)$; large aggregates $\left(D_{\text {eq }}>250 \mu \mathrm{m}\right)$, where $D_{\text {eq }}$ represents the equivalent diameter. For each studied class, aggregates area percentage (Area \%) was calculated, as defined by Amaral and Ferreira (2005). The aggregates total area per volume $(\mathrm{TA} / \mathrm{Vol})$, filaments total length per volume $(\mathrm{TL} /$ Vol), filaments total length per aggregates total area ratio (TL/TA), and filaments total length per mixed liquor suspended solids ratio (TL/MLSS), were also determined according to Mesquita et al. (2010). 
(A)
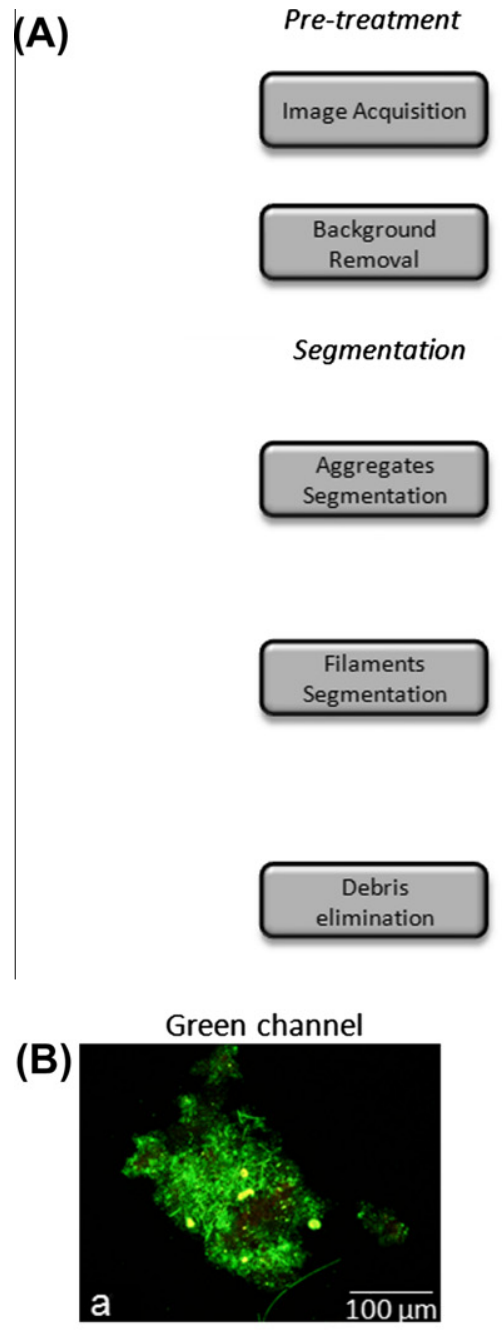
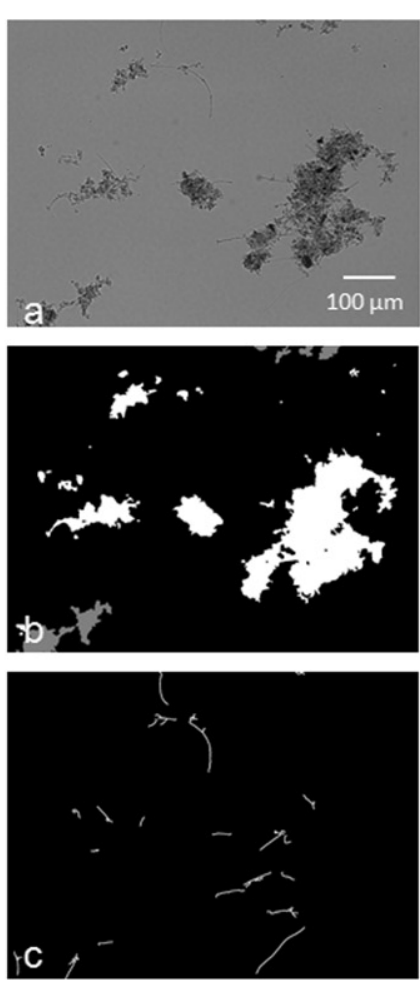

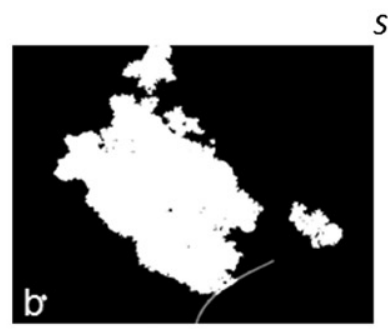

Segmentation and debris elimination

Pre-treatment
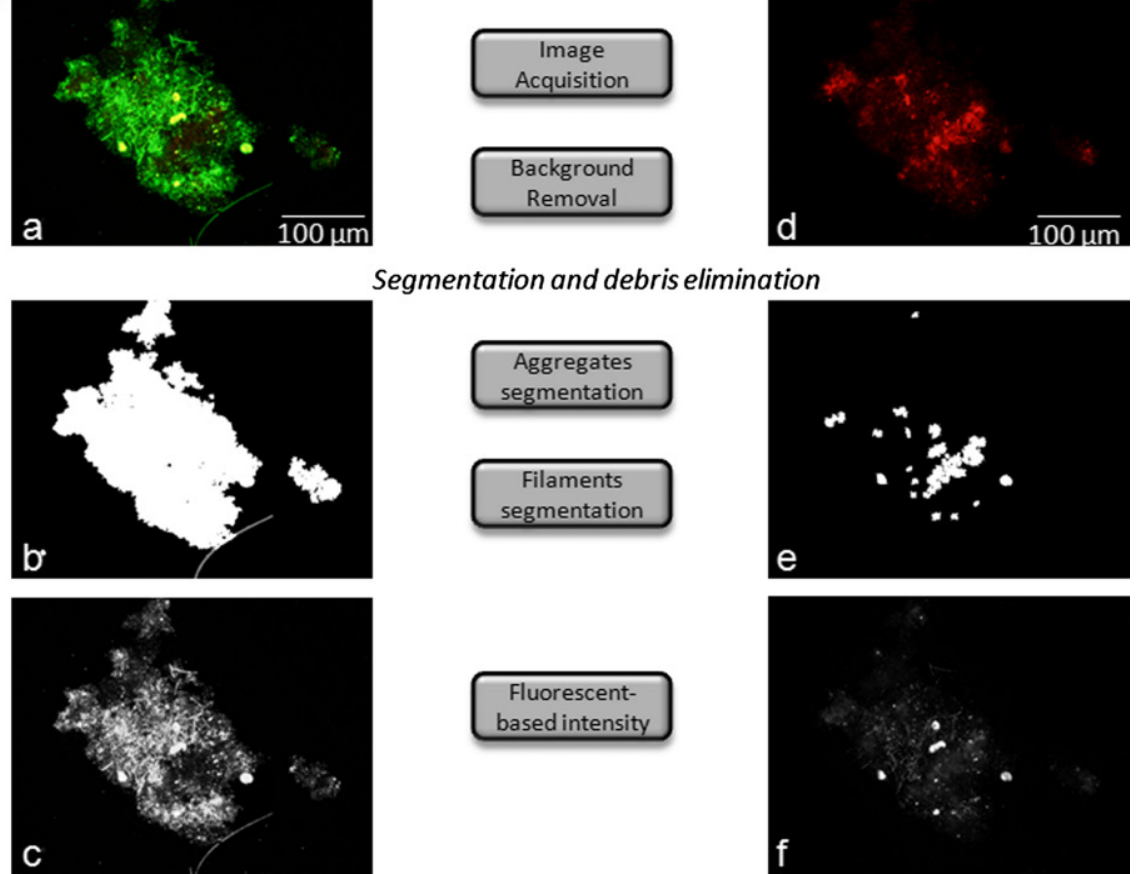

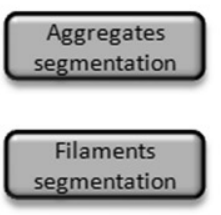

Fluorescentbased intensity

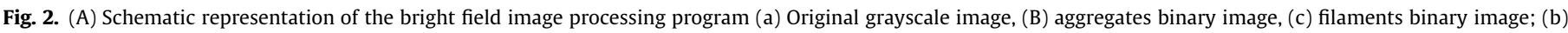

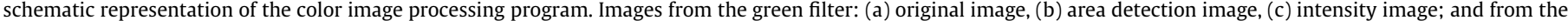

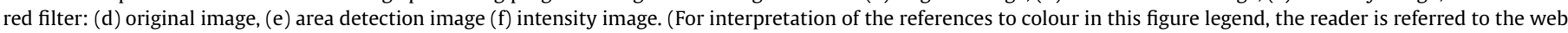
version of this article.)

With respect to the Gram and viability status, the epifluorescence images were segmented to determine the Gram (+), Gram $(-)$, viable and damaged bacteria contents. The ratio between Gram (-) and Gram (+) filamentous bacteria (G_AG/AR) was determined as the corresponding filaments area ratio from the images acquired in fluorescent microscopy of Live BaclightGram stained samples. The ratio between viable and damaged filamentous bacteria (LD_AG/AR) was determined based on the corresponding filaments area ratio from the images acquired in fluorescent microscopy of Live/Dead BacLight bacterial viability stained samples. 


\section{Results and discussion}

\subsection{SVI and MLSS}

The filamentous bulking, PP formation, ZB and NC experiments were performed sequentially, upon the introduction of fresh inoculum between each experiment. Two distinct filamentous bulking conditions were studied, the first one identified as FB1, and the second as FB2. The second filamentous bulking experiment was performed due to the low number of samples provided from the first experiment. Filamentous bulking experiments (FB1 and FB2) were dominated by Thiothrix spp. identified by microscopic inspection of Gram stained samples.

The first step of the data analysis aimed at identifying the period in which each different condition was established. For that purpose, alongside a microscopy inspection, an SVI and MLSS analyses allowed the identification of bulking conditions and PP phenomena recognition. Low SVI values indicate that the sludge is dense and thus the sludge has better settling ability. According to Jenkins et al. (2003), an activated sludge with SVI lower than $120 \mathrm{~mL} \mathrm{~g}^{-1}$ is considered satisfactory and over $150 \mathrm{~mL} \mathrm{~g}^{-1}$ is considered bulking. The determination of bulking conditions was, therefore, based on this $150 \mathrm{~mL} \mathrm{~g}^{-1}$ threshold. Fig. 3 depicts the SVI and MLSS results for each experiment.

Regarding the establishment of bulking conditions, all samples from the first and second periods of filamentous bulking (FB1 and FB2), as well as ZB, presented SVI values higher than $150 \mathrm{~mL} \mathrm{~g}^{-1}$ (with the exception of the last sample of the first filamentous bulking condition with $100 \mathrm{~mL} \mathrm{~g}^{-1}$ ). COD removal percentages of $96 \%$ and $94 \%$ were obtained, in average, during the FB and ZB experiments, respectively. It was also found that filamentous bulking phenomena exhibited higher SVI values (in average) than ZB which is in accordance with the results obtained by Novak et al. (1993). Furthermore, a microscopy inspection allowed identifying a large amount of filamentous bacteria emerging from medium sized flocs in the filamentous bulking experiments, in contrast with the lower filamentous bacteria contents emerging from larger flocs in the ZB experiment (further corroborated by the image analysis results). The presence of filamentous bulking conditions was established given the fact that more than 20 filaments (in average) emerged from each floc, as defined by Jenkins et al. (2003). Furthermore, the image analysis results further corroborated this assumption with a total filaments length of an average 555 for the FB experiment, contrast-

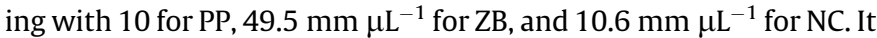
could also be seen, in the current work that the ZB experiment presented a constant MLSS increase, in accordance with high EPS production found in the literature (Novak et al., 1993; Jobbágy et al., 2002). Thus, the comparison of the SVI and MLSS data, alongside the microscopy inspection and image analysis results (referred below) allowed the distinction between filamentous and ZB conditions.

Analyzing the MLSS and SVI results for the PP experiment, it was clear that the SVI remained well below the bulking threshold value, and that the MLSS contents steadily decreased throughout the monitoring period, as expected, given the aggregates washout throughout the experiment.

With respect to the NC experiment, the obtained SVI (lower than $150 \mathrm{~mL} \mathrm{~g}^{-1}$ ), and MLSS values (between 3 and $4 \mathrm{~g} \mathrm{~L}^{-1}$ ) fell within the expected ranges. It should be noticed that, the sharp initial MLSS decrease (from 6-7 to $3-4 \mathrm{~g} \mathrm{~L}^{-1}$ ) was due to the introduction of a highly concentrated inoculum at the beginning of the experiment, and that afterwards the MLSS contents remained approximately constant. COD removal percentages of $92 \%$ and $96 \%$ were obtained, in average, during the PP and NC experiments, respectively.
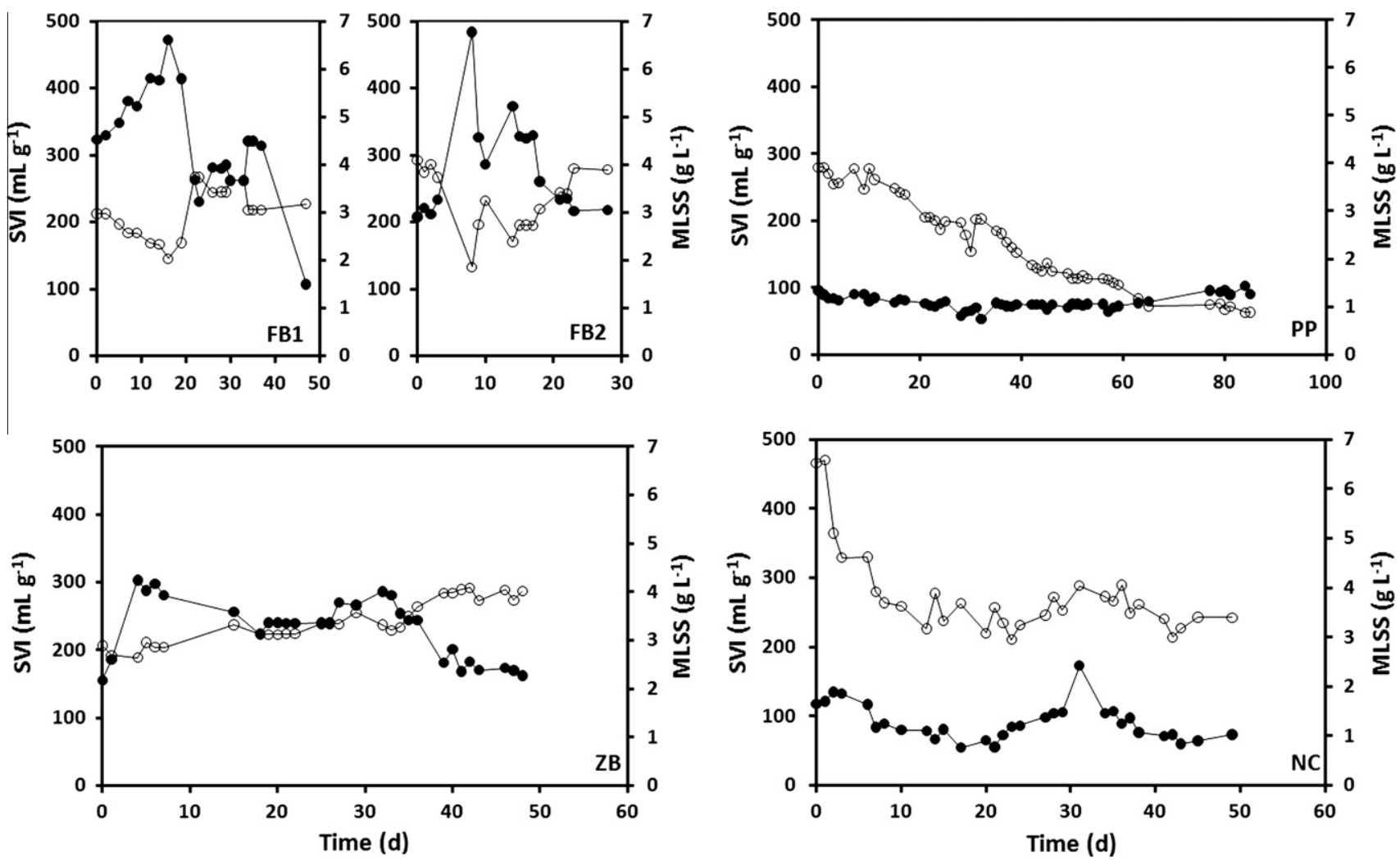

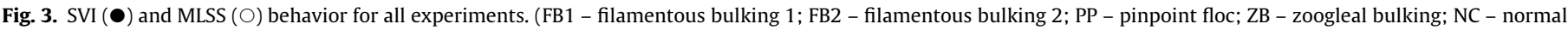
conditions). 


\subsection{Morphological parameters}

Image processing techniques are powerful tools in activated sludge systems for biomass structure inspection, characterization, and quantification. In the present work, the use of such techniques allowed the determination of the biomass structure in terms of the aggregated and filamentous bacteria composition. Furthermore, the size distribution of the aggregated biomass was also studied regarding the percentage of small $\left(D_{\mathrm{eq}}<25 \mu \mathrm{m}\right)$, intermediate $\left(25<D_{\text {eq }}<250 \mu \mathrm{m}\right)$, and large $\left(D_{\text {eq }}>250 \mu \mathrm{m}\right)$ aggregates, as depicted in Fig. 4. During the filamentous bulking experiments (FB1 and FB2), small aggregates prevailed, either dispersed into the mixed liquor, either linked to filamentous bacteria. In contrast, the small aggregates originated by the PP experiment were almost exclusively dispersed in the mixed liquor. Furthermore, throughout this later experiment the intermediate aggregates prevailed with respect to the small flocs, which may be explained by the washout of a considerable percentage of the smaller aggregated biomass. In fact, looking back at the MLSS results (Fig. 3), the strong washout phenomena that occurred during the PP experiment, due to the low compaction and settling abilities of the sludge, could easily have led to the removal of the small aggregates from the reactor. During the ZB experiment, the percentage of large aggregates $\left(D_{\mathrm{eq}}>250 \mu \mathrm{m}\right)$ was higher, on average, than the residual percentages found on the other experiments. In fact, the percentage of large flocs, of $8 \%$, in average, for the ZB experiment, contrasted with the $2 \%$ for $\mathrm{FB}, 1.6 \%$ for $\mathrm{PP}$, and $2.1 \%$ for NC. This result was expected due to the overproduction of exopolymers, thus increasing the aggregates size. Finally, looking at the structure of the biomass during the NC experiments, the results revealed a predominance of the intermediate aggregates regarding the small aggregates, as expected, and residual percentages of large aggregates.
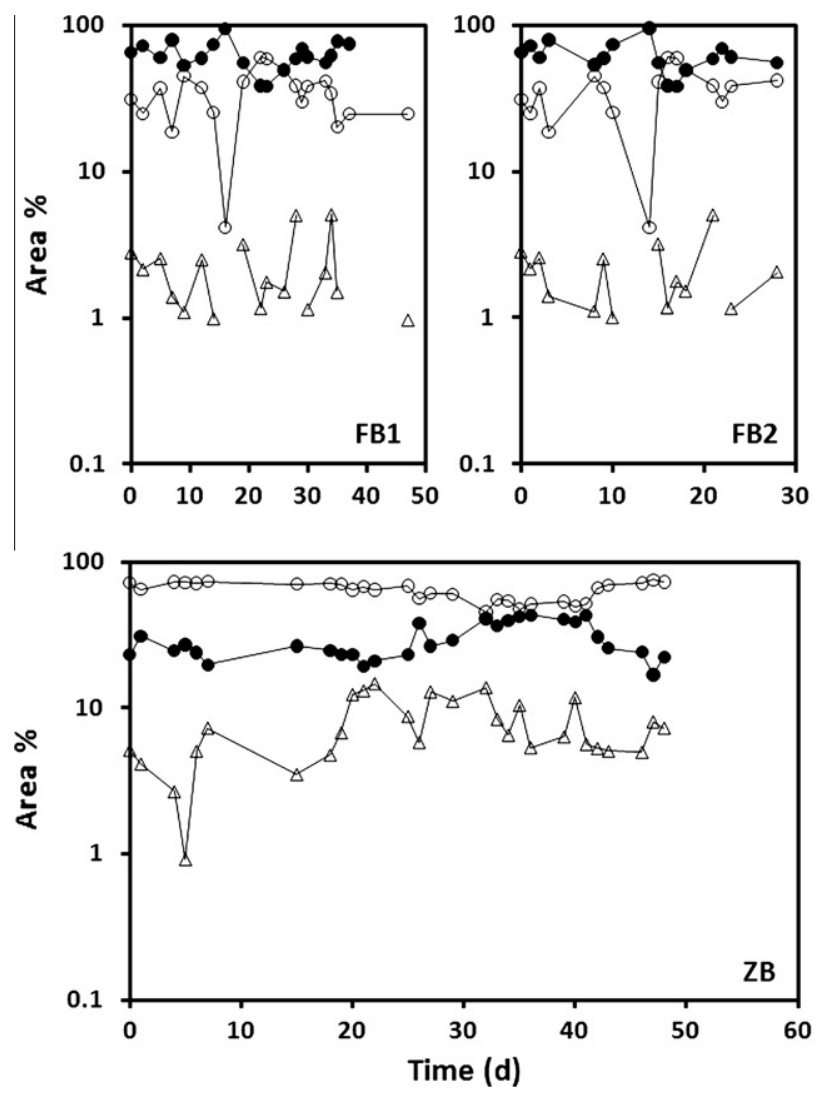

The aggregated biomass (TA/Vol) and filamentous bacteria (TL/ Vol) contents were also studied as shown in Fig. 5a. The filamentous bulking experiments (FB1 and FB2) presented the highest values of the filamentous bacteria contents (TL/Vol), which could be expected given that, by definition, filamentous bulking phenomena occur upon the excessive growth of filamentous bacteria, both inside and extending from the flocs. It could also be seen that the two filamentous bulking events were different from each other, mainly in terms of the aggregated biomass contents, much higher in the second event (FB2). Regarding the ZB experiment the aggregated biomass (TA/Vol) contents increased throughout the experiment, in agreement with the MLSS increase previously discussed. Furthermore, the filamentous bacteria contents (TL/Vol) presented moderate values, although decreasing during the experiment, which could be expected given that the conditions associated with ZB favors the growth of aggregated rather than filamentous bacteria. During the PP period, the aggregated biomass contents (TA/Vol) decreased, as expected given the decrease of the MLSS, configuring biomass washout from the reactor. Furthermore, the filamentous bacteria contents (TL/Vol) remained low, throughout this experiment. Analyzing the biomass structure during the NC experiment, low filaments contents (TL/Vol) were obtained throughout the experiment, whereas the aggregated biomass contents (TA/Vol) fluctuated between moderate values, in accordance with the MLSS behavior.

The ratios between filamentous and aggregated bacteria $(\ln (\mathrm{TL} /$ TA)) and between filamentous bacteria and suspended solids (TL/MLSS) were also studied as presented in Fig. 5b. It seems clear the close relationship between $\ln (\mathrm{TL} / \mathrm{TA})$ and TL/MLSS presenting, in fact, a 0.94 high correlation factor $\left(R^{2}\right)$. The close relationship between these two parameters led to the analysis of the possibility of assessing the TL/MLSS by the $\ln (\mathrm{TL} / \mathrm{TA})$, further described in this section, with the advantage, for activated sludge systems
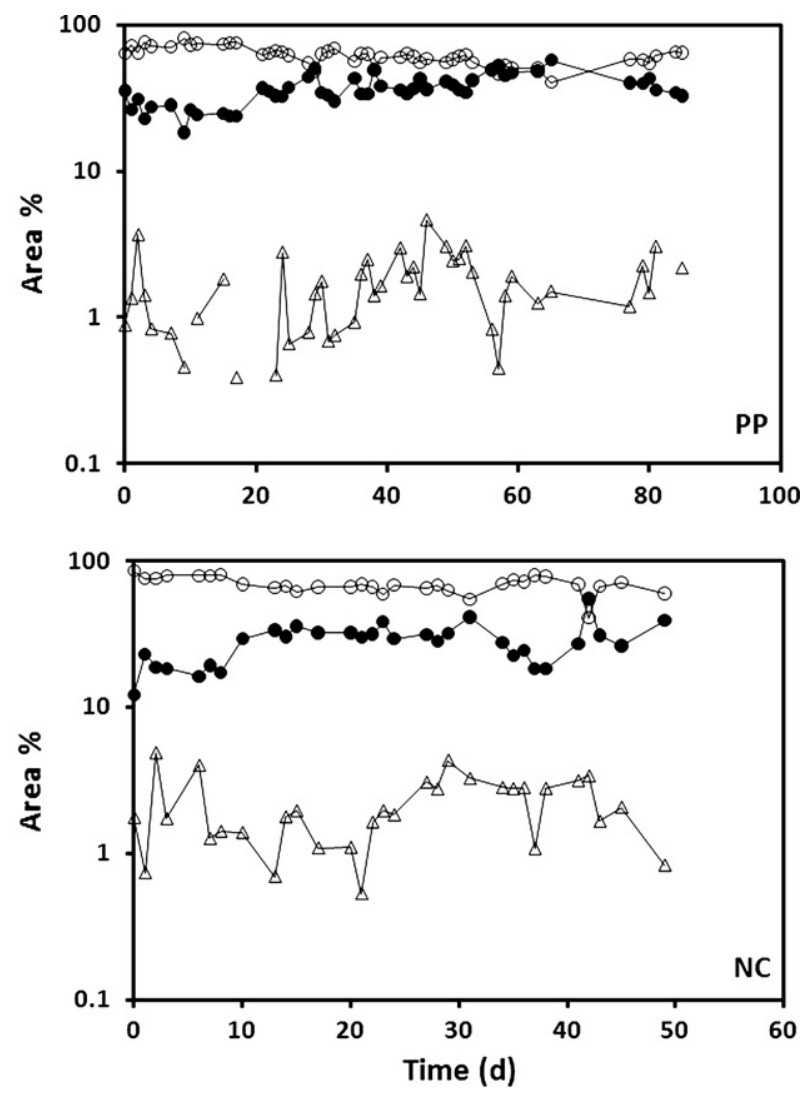

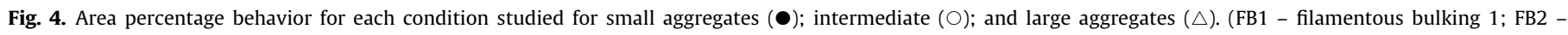
filamentous bulking 2; PP - pinpoint floc; ZB - zoogleal bulking; NC - normal conditions). 

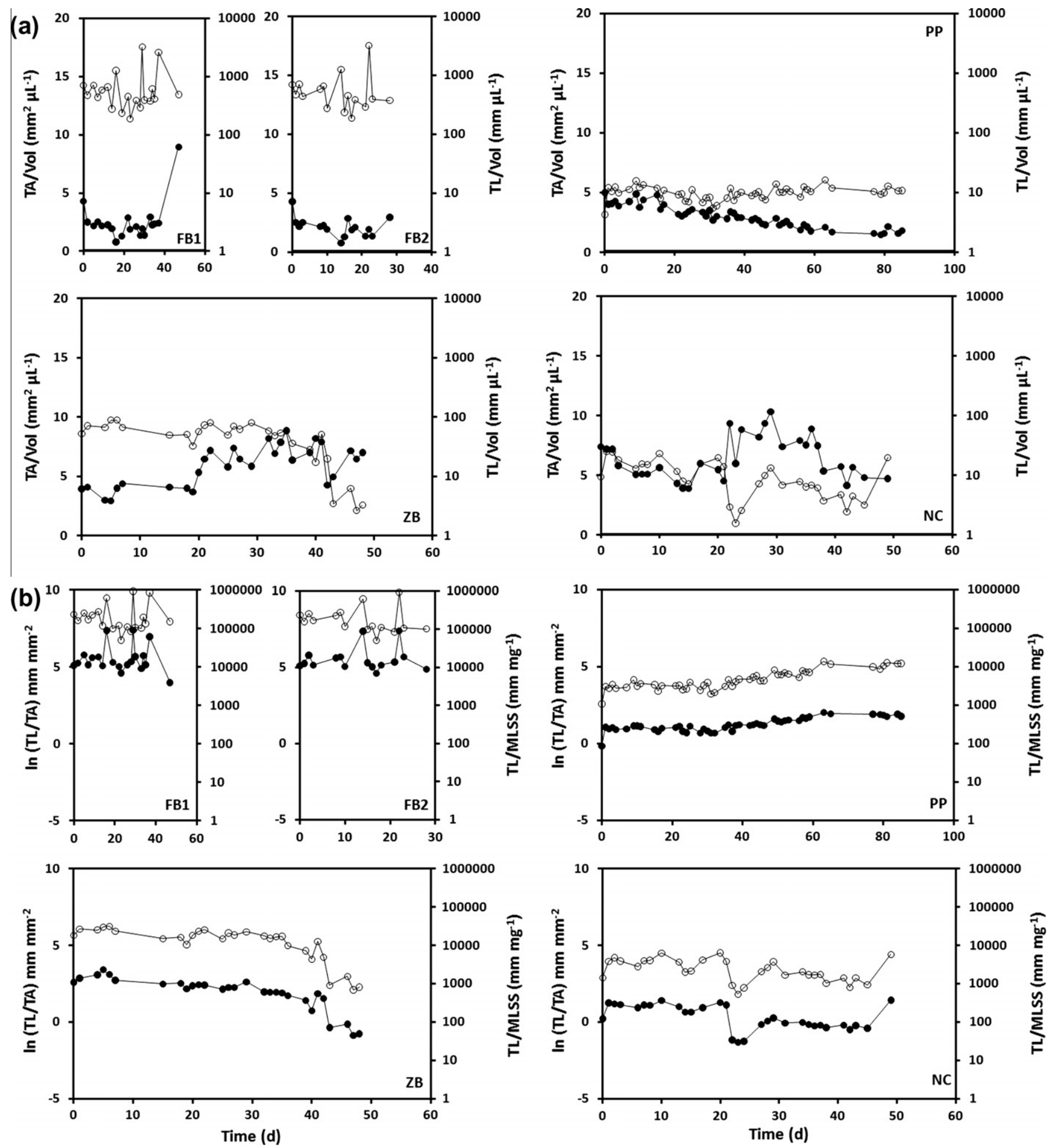

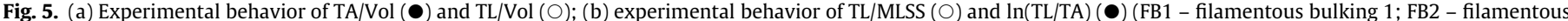
bulking 2; PP - pinpoint floc; ZB - zoogleal bulking; NC - normal conditions).

inspection, that the $\ln (\mathrm{TL} / \mathrm{TA})$ is independent of the suspended solids determination.

The filamentous bulking experiments (FB1 and FB2) presented the highest values for the ratios for the filamentous bacteria contents versus aggregated biomass $(\ln (\mathrm{TL} / \mathrm{TA}))$ and total suspended solids (TL/MLSS). This fact strengthens the identification of these two periods as filamentous bulking phenomena based on the high percentages of filamentous bacteria regarding the overall biomass.
Distinct to the filamentous bulking experiments, the $\ln (\mathrm{TL} / \mathrm{TA})$ and TL/MLSS presented lower values for the ZB experiment decreasing slightly throughout this period (Fig. 5b). These results were expected, since the conditions associated to ZB favors the growth of aggregated biomass contrasting to filamentous bacteria. Regarding the PP experiment, the biomass washout from the reactor led to a very slight growth of the $\ln (\mathrm{TL} / \mathrm{TA})$ and TL/MLSS parameters (although within the range of moderate values) due to the decrease 
of suspended solids. With respect to the NC experiment, and in accordance to the observed low filaments contents, the filamentous bacteria ratios with the aggregated biomass and total suspended solids was kept low throughout the experiment as demonstrated by the $\ln (\mathrm{TL} / \mathrm{TA})$ and TL/MLSS values.

\subsection{SVI assessment}

The image analysis dataset was further used to predict the SVI. In order to do so, SVI values were plotted against the filamentous bacteria contents (TL/Vol) and ratios to the aggregated biomass $(\ln (\mathrm{TL} / \mathrm{TA}))$ and total suspended solids (TL/MLSS). The best results were obtained for the TL/Vol and TL/MLSS correlations, and are presented in Fig. 6a and b. A relationship was found between the SVI and the filamentous bacteria contents (correlation factor $R^{2}$ of 0.83 ), above a TL/Vol threshold value of $7 \mathrm{~mm} \mathrm{\mu L}^{-1}$. Below this value the amount of filamentous bacteria seems not to present significant relevance in SVI values. Furthermore, this correlation is influenced the most by the filamentous and $\mathrm{ZB}$ conditions, given the fact that their values extend throughout the majority of the $Y$ data range. The same analysis was performed for the correlation between SVI and TL/MLSS, which was widely used in previous studies (Lee et al., 1983; Matsui and Yamamoto, 1984; Amaral, 2003; Amaral and Ferreira, 2005). A direct dependence (correlation factor $R^{2}$ of 0.80 ) for TL/MLSS above $2000 \mathrm{~mm} \mathrm{mg}^{-1}$ was achieved, and below this threshold, TL/MLSS values did not seem to influence the SVI. Again, filamentous and ZB conditions, extending throughout the majority of the Y data range, influenced the most the SVI values. Although, past studies have proven the validity of the TL/ MLSS parameter to assess SVI values in filamentous bulking, the present results, encompassing a wider range of conditions, seem to point to the TL/Vol parameter as the most adequate overall. Regarding the adequateness of the $\ln (\mathrm{TL} / \mathrm{TA})$ parameter to capably substitute the TL/MLSS parameter, this could not be fully confirmed given the obtained lower correlation value $R^{2}$ of 0.74 .

\subsection{MLSS assessment}

In the course of this work it was also attempted to elucidate the dependence of MLSS with the TA/Vol parameter, as shown in Fig. 6c. Given the high percentage of filamentous bacteria contributing to MLSS and not account for the TA/Vol (representing solely the aggregates area), all filamentous bulking samples were discarded from this analysis. Therefore, for the PP, ZB and NC MLSS showed a directly dependence (correlation factor $R^{2}$ of 0.83 ) with the aggregated biomass content, but only up to a threshold value of $5 \mathrm{~mm}^{2} \mu \mathrm{L}^{-1}$. Thus, in filamentous bulking conditions or in the case of high aggregated biomass contents it seems not possible to predict MLSS contents from the TA/Vol parameter. Therefore, only for PP, NC and ZB phenomena with low TA/Vol contents, this parameter may be used for the MLSS assessment.

\subsection{Physiological status}

The results from fluorescence microscopy are depicted in Fig. 7, presenting G_AG/AR and LD_AG/AR. A slight difference between the filamentous bulking experiments 1 and 2 was detected. Although in both cases Gram (-) bacteria predominated, during FB1 their number varied from 10- to 100 -fold the Gram (+) bacteria, whereas in FB2 their number was smaller, varying from 1 to 10 -fold. Furthermore, regarding the filamentous bacteria viability, and although the vast majority of cells were viable, FB1 and FB2 also differed considerably. The two periods presented opposite tendencies with increasing filamentous bacteria viability throughout FB1 and decreasing filamentous bacteria viability throughout FB2. Regarding the ZB experiment Gram (-) bacteria predominated, varying from 1 to 100 -fold whereas the filamentous bacteria viability fluctuated throughout the experiment, although maintaining a large majority of viable cells. In the pinpoint experiment, a shift on the filamentous bacteria community was observed from initially predominant Gram (-) bacteria towards a majority of
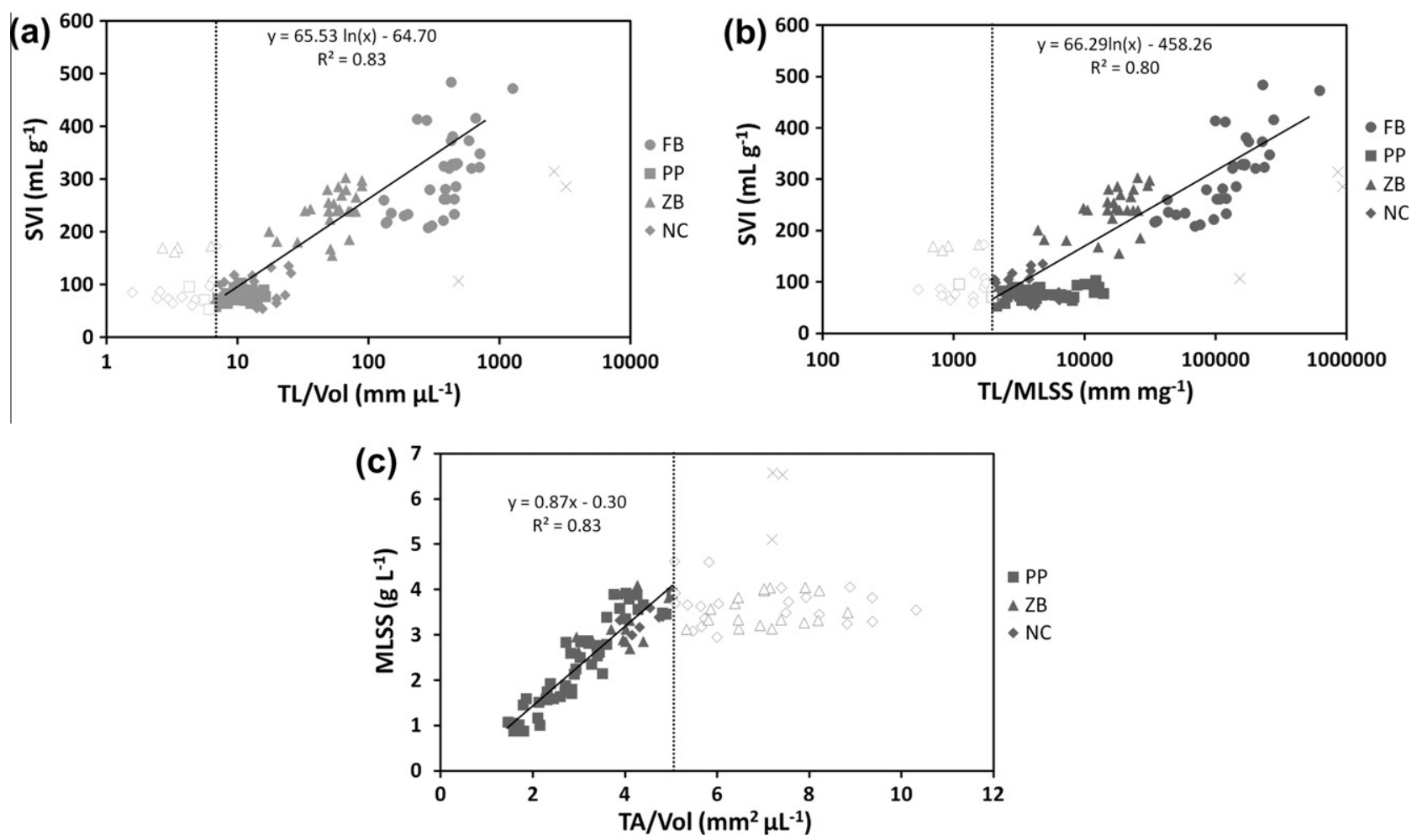

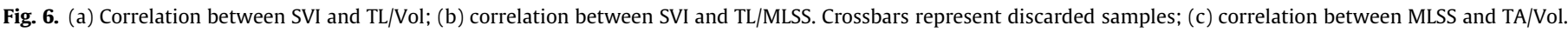
Crossbars represent discarded samples. (FB - filamentous bulking; PP - pinpoint flocs; ZB - zoogleal bulking; NC - normal conditions). 

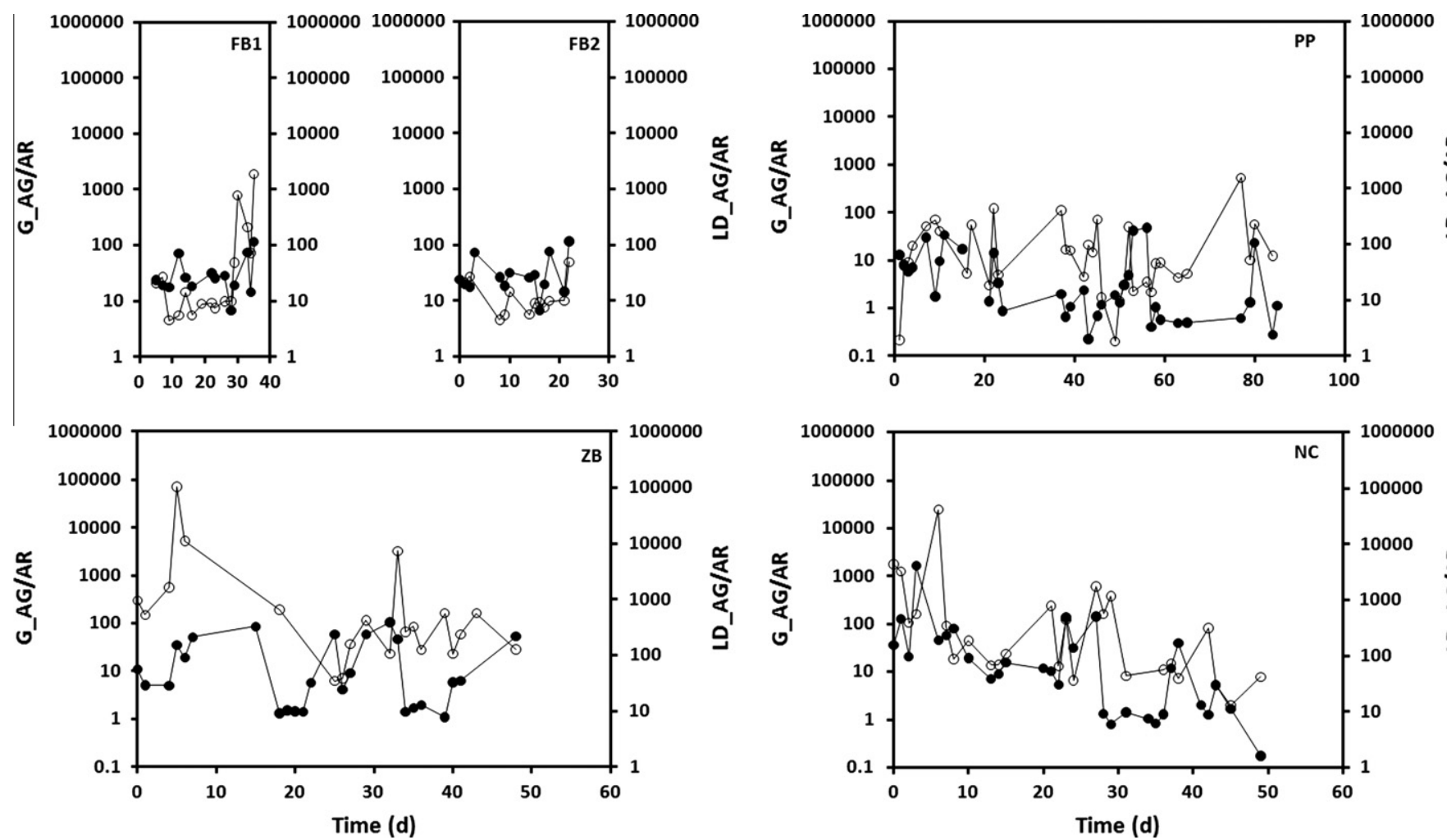

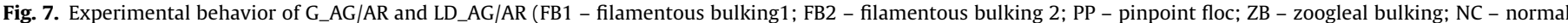
conditions).

Gram (+) bacteria at the end of the experiment. Regarding the filamentous bacteria viability no clear trend was observed throughout the experiment, maintaining a large majority of viable cells. The NC experiment started with the predominance of filamentous Gram (-) bacteria that progressively shifted towards the equilibrium with the Gram $(+)$ bacteria at the end of the experiment. Regarding the filamentous bacteria viability a decreasing trend in the filamentous bacteria viability was noticed throughout the experiment, although maintaining a large majority of viable cells.

\section{Conclusions}

Image analysis parameters were used to elucidate several disturbances that may occur in an activated sludge system, and the possibility of measuring two of the most important operational parameters (SVI and MLSS) for biomass characterization. During both filamentous bulking experiments the majority of the aggregated biomass formed small aggregates, either dispersed in the mixed liquor, either attached to filamentous bacteria. Furthermore, filamentous bulking presented the largest filamentous bacteria contents, as well as their ratio versus aggregated bacteria and suspended solids. Differences between the filamentous bacteria composition and viability from filamentous bulking experiments 1 and 2 were also detected. Although in both cases viable Gram (-) bacteria predominated, FB1 presented a larger Gram (-) fraction with increasing viability throughout the experiment, opposite to the decreasing trend in FB2. The ZB experiment presented the largest contents of large aggregates alongside the increase of both the aggregated biomass and MLSS. The filamentous bacteria contents as well as their ratio versus aggregated bacteria and suspended solids presented a decreasing trend throughout this period, since the conditions associated to ZB favors the growth of aggregated biomass contrasting to filamentous bacteria. Furthermore it was detected a predominance of Gram (-) and viable bacteria.
Regarding the PP experiment, it was possible to observe a biomass washout phenomenon leading to the decrease of biomass contents mirrored by the MLSS and aggregated biomass behaviors, which, in turn led to a continuous growth of the $\ln (\mathrm{TL} / \mathrm{TA})$ and TL/MLSS parameters (although within the range of moderate values). During this experiment, a large majority of viable cells was observed although the filamentous bacteria community shifted from initially predominant Gram (-) bacteria towards a majority of Gram (+) bacteria at the end of the experiment. The biomass structure under NC showed a predominance of intermediate flocs, low filamentous bacteria contents and respective ratios versus aggregated bacteria and suspended solids. Furthermore, it was noticed a shift from an initial clear predominance of filamentous Gram (-) bacteria towards the equilibrium with the Gram (+) bacteria at the end of the experiment. Although slightly decreasing throughout the experiment, a large majority of viable cells was maintained.

Regarding the SVI assessment, a relationship (correlation factor $R^{2}$ of 0.83 ) between the SVI and the filamentous bacteria contents was found, for $\mathrm{TL} / \mathrm{Vol}$ values above $7 \mathrm{~mm} \mu \mathrm{L}^{-1}$. Although, past studies have proven the validity of the TL/MLSS parameter to assess SVI values in filamentous bulking, the present results, encompassing a wider range of conditions, seem to point to the TL/Vol parameter as the most adequate overall. In the current work, the assessment of MLSS by image analysis was limited to PP, NC and ZB phenomena with low TA/Vol contents $\left(5 \mathrm{~mm}^{2} \mu \mathrm{L}^{-1}\right)$, thus excluding filamentous bulking and high aggregated biomass contents systems. In conclusion it may be inferred that the image analysis methodology clearly revealed the nature of each studied condition during this work.

\section{Acknowledgments}

The authors acknowledge the financial support to D.P.M. through the Grant SFRH/BD/32329/2006 and the project PTDC/ 
EBB-EBI/103147/2008 provided by Fundação para a Ciência e a Tecnologia.

\section{References}

Amaral, A.L., 2003. Image Analysis in Biotechnological Processes: Applications to Wastewater Treatment. PhD Thesis, University of Minho, Braga, Portugal. <http://hdl.handle.net/1822/4506>.

Amaral, A.L., Ferreira, E.C., 2005. Activated sludge monitoring of a wastewater treatment plant using image analysis and partial least squares regression. Anal. Chim. Acta 544, 246-253.

Andreadakis, A., 1993. Physical and chemical properties of activated sludge flocs. Water Res. 27, 1707-1714.

APHA, 1989. Standard Methods for the Examination of Water and Wastewater, 17th ed. American Public Health Association, Washington, DC

Arelli, A., Luccarini, L., Madoni, P., 2009. Application of image analysis in activated sludge to evaluate correlations between settleability and features of flocs and filamentous species. Water Sci. Technol. 59, 2029-2036.

Barbusinski, K., Koscielniak, H., 1995. Influence of substrate loading intensity on floc size in activated sludge process. Water Res. 29, 1703-1710.

Boulos, L., Prévost, M., Barbeau, B., Coallier, J., Desjardins, R., 1999. LIVE/DEAD ${ }^{\circledR}$ BacLight ${ }^{\mathrm{TM}}$ : application of a new rapid staining method for direct enumeration of viable and total bacteria in drinking water. J. Microbiol. Methods 37, 77-86.

Cenens, C., Van Beurden, K.P., Jenné, R., Van Impe, J.F., 2002. On the development of a novel image analysis technique to distinguish between flocs and filaments in activated sludge images. Water Sci. Technol. 46 (1-2), 381-387.

da Motta, M., Pons, M.N., Roche, N., 2002. Study of filamentous bacteria by image analysis and relation with settleability. Water Sci. Technol. 46 (1-2), 363-369.

Eikelboom, D.H., 2000. Process Control of Activated Sludge Plants by Microscopic Investigation, first ed. IWA Publishing, London.

Foster, S., Snape, J.R., Lappin-Scott, H.M., Porter, J., 2002. Simultaneous fluorescent gram staining and activity assessment of activated sludge bacteria. Appl. Environ. Microbiol. 68, 4772-4779.

Grijspeerdt, K., Verstraete, W., 1997. Image analysis to estimate the settleability and concentration of activated sludge. Water Res. 31, 1126-1134.

Invitrogen Molecular Probes, 2004. LIVE/DEAD BacLight ${ }^{\mathrm{TM}}$ Bacterial Viability Kits. Manuals and Product Inserts. <http://probes.invitrogen.com/media/pis/ mp07007.pdf>.

Jenkins, D., Richard, M.G., Daigger, G., 2003. Manual on the Causes and Control of Activated Sludge Bulking, Foaming and Other Solids Separation Problems. Lewis Publishing, Boca Raton, FL.

Jenné, R., Banadda, E.N., Gins, G., Deurinck, J., Smets, I.Y., Geeraerd, A.H., Van Impe, J.F., 2006. Use of image analysis for sludge characterization: studying the relation between floc shape and sludge settleability. Water Sci. Technol. 54 (1), 167-174.
Jin, B., Wilén, B.M., Lant, P., 2004. Impacts of morphological, physical and chemical properties of sludge flocs on dewaterability of activated sludge. Chem. Eng. J. 98, $115-126$.

Jin, B., Wilén, B.M., Lant, P., 2003. A comprehensive insight into floc characteristics and their impact on compressibility and settleability of activated sludge. Chem. Eng. J. 95, 221-234

Jobbágy, A., Literáthy, B., Tardy, G., 2002. Implementation of glycogen accumulating bacteria in treating nutrient-deficient wastewater. Water Sci. Technol. 46 (1-2), 185-190.

Lee, S.E., Koopman, B., Bode, H., Jenkins, D., 1983. Evaluation of alternative sludge settleability indexes. Water Res. 17, 1421-1426.

Li, D., Ganczarczyk, J., 1987. Stroboscopic determination of settling velocity, size and porosity of activated sludge flocs. Water Res. 21, 257-262.

Li, D., Ganczarczyk, J., 1991. Size distribution of activated sludge flocs. J. Water Pollut. Control Fed. 63, 806-814.

Li, X.Y., Yang, S.F., 2007. Influence of loosely bound extracellular polymeric substances (EPS) on the flocculation, sedimentation and dewaterability of activated sludge. Water Res. 41, 1022-1030.

Liao, B.Q., Droppo, I.G., Leppard, G.G., Liss, S.N., 2006. Effect of solids retention time on structure and characteristics of sludge flocs in sequencing batch reactors. Water Res. 40, 2583-2591.

Martins, A.M.P., Pagilla, K., Heijnen, J.J., van Loosdrecht, M.C.M., 2004. Filamentous bulking sludge - a critical review. Water Res. 38, 793-817.

Mesquita, D.P., Dias, O., Amaral, A.L., Ferreira, E.C., 2009a. Monitoring of activated sludge settling ability through image analysis: validation on full-scale wastewater treatment plants. Bioprocess Biosyst. Eng. 32, 361-367.

Mesquita, D.P., Dias, O., Amaral, A.L., Ferreira, E.C., 2010. A comparison between bright field and phase contrast image analysis techniques in activated sludge morphological characterization. Microsc. Microanal. 16, 166-174.

Mesquita, D.P., Dias, O., Dias, A.M.A., Amaral, A.L., Ferreira, E.C., 2009b. Correlation between sludge settleability and image analysis information using partial least squares. Anal. Chim. Acta 642, 94-101.

Matsui, S. Yamamoto, R, 1984. The use of color TV technique for measuring filament length and investigating sludge bulking causes. Water Sci. Technol. 16 (10-11), 69-81.

Novak, L., Larrea, L., Wanner, J., Garcia-Heras, J.L., 1993. Non-filamentous activated sludge bulking in a laboratory scale system. Water Res. 27, 1339-1346.

Peng, Y., Gao, C., Wang, S., Ozaki, M., Takigawa, A., 2003. Non-filamentous sludge bulking caused by a deficiency of nitrogen in industrial wastewater treatment. Water Sci. Technol. 47 (11), 289-295.

Schuler, A.J., Jassby, D., 2007. Filament content threshold for activated sludge bulking: artifact or reality? Water Res. 41, 4349-4356.

Urbain, V., Block, J.C., Manem, D.J., 1993. Biofloculation in activated sludge: an analytical approach. Water Res. 27, 829-838.

Wilén, B.M., Jin, B., Lant, P., 2003. The influence of key chemical constituents in activated sludge on surface and flocculating properties. Water Res. 37, $2127-$ 2139. 